\title{
Parameter-Uniform Improved Hybrid Numerical Scheme for Singularly Perturbed Problems with Interior Layers
}

\section{Kaushik Mukherjee}

\author{
Department of Mathematics, Indian Institute of Space Science and Technology \\ 695547 Thiruvananthapuram, Kerala, India \\ E-mail(corresp.): mathkaushik@gmail.com, kaushik@iist.ac.in
}

Received December 16, 2016; revised January 13, 2018; accepted January 19, 2018

\begin{abstract}
In this paper, we consider a class of singularly perturbed convectiondiffusion boundary-value problems with discontinuous convection coefficient which often occur as mathematical models for analyzing shock wave phenomena in gas dynamics. In general, interior layers appear in the solutions of this class of problems and this gives rise to difficulty while solving such problems using the classical numerical methods (standard central difference or standard upwind scheme) on uniform meshes when the perturbation parameter $\varepsilon$ is small. To achieve better numerical approximation in solving this class of problems, we propose a new hybrid scheme utilizing a layer-resolving piecewise-uniform Shishkin mesh and the method is shown to be $\varepsilon$ uniformly stable. In addition to this, it is proved that the proposed numerical scheme is almost second-order uniformly convergent in the discrete supremum norm with respect to the parameter $\varepsilon$. Finally, extensive numerical experiments are conducted to support the theoretical results. Further, the numerical results obtained by the newly proposed scheme are also compared with the hybrid scheme developed in the paper [Z.Cen, Appl. Math. Comput., 169(1): 689-699, 2005]. It shows that the current hybrid scheme exhibits a significant improvement over the hybrid scheme developed by Cen, in terms of the parameter-uniform order of convergence.
\end{abstract}

Keywords: singularly perturbed boundary-value problem, interior layer, numerical scheme, piecewise-uniform Shishkin mesh, uniform convergence.

AMS Subject Classification: 65L10; CR G1.7.

\section{Introduction}

In this paper, a class of singularly perturbed boundary-value problems (BVPs) with discontinuous convection coefficient is considered on the unit interval

Copyright (C) 2018 The Author(s). Published by VGTU Press

This is an Open Access article distributed under the terms of the Creative Commons Attribution License (http://creativecommons.org/licenses/by/4.0/), which permits unrestricted use, distribution, and reproduction in any medium, provided the original author and source are credited. 
$\Omega=(0,1)$ and the convection coefficient is assumed to be discontinuous at a single point $x=\xi \in \Omega$. Let $\Omega^{-}=(0, \xi), \Omega^{+}=(\xi, 1)$ and the jump of a function $w$, denoted by $[w]$, across the point of discontinuity $x=\xi$ is defined by $[w](\xi)=w\left(\xi^{+}\right)-w\left(\xi^{-}\right)$, where $w\left(\xi^{ \pm}\right)=\lim _{x \rightarrow \xi \pm 0} w(x)$. The model problem is then described as follows:

$$
\left\{\begin{array}{l}
L_{\varepsilon} u(x) \equiv \varepsilon u^{\prime \prime}(x)+a(x) u^{\prime}(x)-b(x) u(x)=f(x), \quad x \in \Omega^{-} \cup \Omega^{+}, \\
u(0)=u_{0}, \quad u(1)=u_{1},
\end{array}\right.
$$

where $0<\varepsilon \ll 1$ is a small parameter. Here, the convection coefficient $a$, the reaction term $b$ and the source term $f$ satisfy the following assumptions

$$
\left\{\begin{array}{l}
b(x) \geq 0 \quad \text { on } \bar{\Omega}, \quad|[a](\xi)| \leq C, \quad|[f](\xi)| \leq C, \\
-\alpha_{1}^{*}<a(x)<-\alpha_{1}<0, \quad \text { for } x<\xi, \\
\alpha_{2}^{*}>a(x)>\alpha_{2}>0, \quad \text { for } x>\xi,
\end{array}\right.
$$

and the solution $u$ satisfies the following interface conditions

$$
[u](\xi)=0, \quad\left[\frac{d u}{d x}\right](\xi)=0 .
$$

The BVP (1.1)-(1.2) admits a unique solution $u \in \mathcal{C}^{1}(\Omega) \cap \mathcal{C}^{2}\left(\Omega^{-} \cup \Omega^{+}\right)$ (see [2]). Throughout the paper, we assume that $a, b, f \in \mathcal{C}^{3}\left(\Omega^{-} \cup \Omega^{+}\right)$so that these functions can be extended into $\overline{\Omega^{-}}$and $\overline{\Omega^{+}}$in $\mathrm{C}^{3}$. In general, due to the particular pattern of the convection coefficient $a$ on the either side of the point of discontinuity, the solution possesses strong interior layers of width $O(\varepsilon)$ in the neighborhood of the point $x=\xi$.

Convection-diffusion equations of the form (1.1)-(1.2) with discontinuous convection coefficient has application for numerous physical problems in engineering and applied mathematics, which typically includes linearized steady state viscous Burgers' equation for studying shock wave phenomena in gas dynamics. The special characteristic of singularly perturbed differential equations even with continuous data is that due to the presence of layers in the exact solution, which are certain narrow regions in which the behaviour of the solution changes very rapidly, the classical numerical methods usually yield unsatisfactory numerical results on uniform meshes, whenever the perturbation parameter $\varepsilon$ tends to zero. In order to overcome this difficulty, parameteruniform numerical methods are designed so that for all $N \geq N_{0}$, where $N$ is the number of mesh-intervals and $N_{0}$ is some positive integer independent of $\varepsilon$, the error constant and order of convergence measured in the discrete supremum norm are independent of $\varepsilon$. In this context, the fitted mesh parameter-uniform numerical methods (see the books $[6,11]$ and the survey article [5]), which utilize special layer-adapted meshes, are found to be satisfactory and popular techniques.

Over the last few years, several researchers developed the fitted mesh methods for solving singularly perturbed problems with non-smooth data, one can refer the articles $[1,2,4,10,12]$ for the stationary case and $[7,8,9]$ for the nonstationary case. However, it is worth mentioning that the hybrid numerical 
scheme proposed by Cen in [1], is an well-known fitted mesh method for solving singularly perturbed BVPs of the form (1.1)-(1.2) with discontinuous convection coefficient and the method is almost second-order accurate throughout the domain $[0,1]$ provided the perturbation parameter $\varepsilon$ satisfies $\varepsilon \ll N^{-1}$, otherwise the method is at worst first-order uniformly convergent with respect to $\varepsilon$ in the discrete supremum norm (see the detailed discussion in Section 6). Therefore, it is quite natural to ask whether one can design a hybrid scheme which attains an improvement with respect to the $\varepsilon$-uniform order of convergence, compared to the above mentioned hybrid scheme.

The prime objective of this article is to develop a new hybrid scheme on a piecewise-uniform Shishkin mesh for solving the singularly perturbed BVPs of the form (1.1)-(1.2) so that the method is at least second-order uniformly convergent with respect to $\varepsilon$ in the discrete supremum norm. The proposed hybrid scheme consists of a suitable combination of a second-order accurate modified central difference scheme and the midpoint upwind scheme. The advantage of such blending in the proposed scheme lies in the fact that it overcomes the limitation of the standard central difference scheme (i.e., related to the loss in stability for sufficiently small $\varepsilon$ ) and that of the standard upwind scheme (i.e., related to the lower-order accuracy), while solving the singularly perturbed convection-diffusion BVPs. It is observed that the finite difference operator $L_{\varepsilon}^{N}$ associated with the proposed numerical scheme does not satisfy the monotonicity property. To overcome this difficulty, we show that it is possible to transform the system of equations (described in (3.1)) into a tridiagonal system of equations and the finite difference operator $L_{h y b}^{N}$ associated with the new system of equations does satisfy the desirable monotonicity property. Hereby, it is shown that the proposed scheme is $\varepsilon$-uniformly stable, which leads to the uniqueness of the numerical solution. Apart from this, we prove that the newly proposed scheme is almost second-order (up to the logarithmic factor) accurate in the discrete supremum norm throughout the domain $[0,1]$ and it does not require the restrictive assumption $\varepsilon \ll N^{-1}$ to be held.

We organize the rest of the paper as follows. In Section 2, we present a priori bounds on the analytical solution and its derivatives. Section 3 describes the Shishkin mesh and provides the detail construction of the proposed hybrid finite difference scheme. In Section 4 , we discuss the $\varepsilon$-uniform stability of the proposed hybrid scheme. Afterwards in Section 5, we prove the main convergence result related to the $\varepsilon$-uniform error bound of the proposed numerical scheme. Finally, we carry out the extensive numerical experiments in Section 6 to validate the theoretical results and also to demonstrate the efficiency and accuracy of the proposed scheme, we compare the numerical results obtained by the proposed hybrid scheme with the hybrid scheme developed in [1]. We end up this section by stating observations about the newly proposed scheme with concluding remarks.

Throughout the paper $C$ denotes a generic positive constant that is independent of the perturbation parameter $\varepsilon$ and the number of mesh-intervals $N$. Here, $\|\cdot\|_{D}$ stands for the supremum norm, which is defined by

$$
\|g\|_{D}=\sup _{\xi \in D}|g(\xi)|
$$


for a function $g$ defined on some domain $D$.

\section{Bounds on the analytical solution and its derivatives}

In this section, we provide a priori bounds on the analytical solution of the BVP (1.1)-(1.2) and its derivatives. These bounds reflect the asymptotic behavior of the analytical solution with respect to the singular perturbation parameter $\varepsilon$, and are also essential to establish the $\varepsilon$-uniform error estimate stated in Theorem 2.

Theorem 1. The solution $u$ of the BVP (1.1)-(1.2) can be decomposed as $u=v+w$, where the smooth component $v$ and the non-smooth component $w$ respectively satisfy

$$
L_{\varepsilon} v(x)=f(x) \quad \text { and } \quad L_{\varepsilon} w(x)=0, \quad x \in \Omega^{-} \cup \Omega^{+}
$$

with suitable boundary conditions such that for integers $0 \leq k \leq 4$, the smooth component $v$ and the non-smooth component $w$ respectively, satisfy the following bounds

$$
\begin{aligned}
& \|v\|_{\bar{\Omega}} \leq C, \quad\left\|v^{k}\right\|_{\Omega^{-} \cup \Omega^{+}} \leq C\left(1+\varepsilon^{3-k}\right), \\
& \left|w^{k}(x)\right| \leq \begin{cases}C\left[\varepsilon^{-k} \exp \left(-(\xi-x) \alpha_{1} / \varepsilon\right)\right], & x \in \Omega^{-}, \\
C\left[\varepsilon^{-k} \exp \left(-(x-\xi) \alpha_{2} / \varepsilon\right)\right], & x \in \Omega^{+} .\end{cases}
\end{aligned}
$$

Proof. The proof follows from [ [2], Lemma 4].

\section{Numerical approximation}

This section describes the piecewise-uniform Shishkin mesh resolving the interior layers and introduces the proposed finite difference scheme which is used to discretize the BVP (1.1)-(1.2).

\subsection{Piecewise-uniform Shishkin mesh}

Consider the domain $\bar{\Omega}=[0,1]$ and let $N \geq 16$ be an even positive integer. Since the BVP (1.1)-(1.2) has interior layers at $x=\xi$, we construct a piecewiseuniform Shishkin mesh by subdividing the domain $\bar{\Omega}$ into four subintervals as

$$
\bar{\Omega}=\left[0, \xi-\sigma_{1}\right] \cup\left[\xi-\sigma_{1}, \xi\right] \cup\left[\xi, \xi+\sigma_{2}\right] \cup\left[\xi+\sigma_{2}, 1\right],
$$

where the transition parameters $\sigma_{1}$ and $\sigma_{2}$ are defined as

$$
\sigma_{1}=\min \left\{\frac{\xi}{2}, \sigma_{0} \varepsilon \ln N\right\}, \quad \sigma_{2}=\min \left\{\frac{1-\xi}{2}, \sigma_{0} \varepsilon \ln N\right\} .
$$

Here, we choose $\sigma_{0}=2 / \gamma$, where $\gamma$ is a positive constant. Note that this mesh will be uniform if $\sigma_{1}=\xi / 2, \sigma_{2}=(1-\xi) / 2$. On each subinterval, we place a uniform mesh with $N / 4$ mesh-intervals such that

$$
\Omega^{N}=\left\{x_{i}: 1 \leq i \leq N / 2-1\right\} \bigcup\left\{x_{i}: N / 2+1 \leq i \leq N-1\right\},
$$


which denotes the set of interior points of the mesh. Then, clearly $x_{N / 2}=\xi$ and $\bar{\Omega}^{N}=\left\{x_{i}\right\}_{0}^{N}$. Let $h_{i}=x_{i}-x_{i-1}$ denote the step size and also let $\widehat{h}_{i}=$ $h_{i}+h_{i+1}$, for $i=1, \ldots, N-1$. Further, we denote the mesh width $h_{i}$ as follows:

$$
h_{i}= \begin{cases}H_{(l)}=4\left(\xi-\sigma_{1}\right) / N, & \text { for } i=1, \ldots, N / 4 \\ h_{(l)}=4 \sigma_{1} / N, & \text { for } i=N / 4+1, \ldots, N / 2 \\ h_{(r)}=4 \sigma_{2} / N, & \text { for } i=N / 2+1, \ldots, 3 N / 4 \\ H_{(r)}=4\left(1-\xi-\sigma_{2}\right) / N, & \text { for } i=3 N / 4+1, \ldots, N\end{cases}
$$

\subsection{Hybrid numerical scheme}

For a given mesh function $Z\left(x_{i}\right)=Z_{i}$, we define the forward, backward and the second-order finite difference operators $D^{+}, D^{-}$and $\delta^{2}$ respectively by

$$
D^{+} Z_{i}=\frac{Z_{i+1}-Z_{i}}{h_{i+1}}, D^{-} Z_{i}=\frac{Z_{i}-Z_{i-1}}{h_{i}} \text { and } \delta^{2} Z_{i}=\frac{2\left(D^{+} Z_{i}-D^{-} Z_{i}\right)}{\widehat{h}_{i}}
$$

and the modified central difference operator $D^{\star}$ by

$$
D^{\star} Z_{i}=\left(\frac{h_{i}}{\widehat{h}_{i}}\right) D^{+} Z_{i}+\left(\frac{h_{i+1}}{\widehat{h}_{i}}\right) D^{-} Z_{i}
$$

Also we define $Z_{i \pm \frac{1}{2}}=\left(Z_{i}+Z_{i \pm 1}\right) / 2$. For the discretization of the BVP (1.1)(1.2), we now describe the proposed hybrid numerical scheme which consists of a modified central difference scheme when $\varepsilon>2\|a\| N^{-1}$ and a proper combination of the midpoint upwind scheme in the outer regions $\left(0, \xi-\sigma_{1}\right],\left[\xi+\sigma_{2}, 1\right)$ and the modified central difference scheme in the interior layer regions $(\xi-$ $\left.\sigma_{1}, \xi\right),\left(\xi, \xi+\sigma_{2}\right)$, when $\varepsilon \leq 2\|a\| N^{-1}$. At the point of discontinuity, secondorder one-sided difference approximations are used. The numerical scheme then takes the following form:

$$
\left\{\begin{array}{rr}
\varepsilon \delta^{2} U_{i}+a_{i} D^{\star} U_{i}-b_{i} U_{i}=f_{i}, \quad \text { for } i=N / 4+1, \ldots, N / 2-1, N / 2+1, \ldots, & 3 N / 4-1, \\
\varepsilon \delta^{2} U_{i}+a_{i} D^{\star} U_{i}-b_{i} U_{i}=f_{i}, & \text { for } i=1, \ldots, N / 4,3 N / 4, \ldots, N-1, \text { and } \\
& \text { when } \varepsilon>2\|a\| N^{-1}, \\
\varepsilon \delta^{2} U_{i}+a_{i-\frac{1}{2}} D^{-} U_{i}-b_{i-\frac{1}{2}} U_{i-\frac{1}{2}}=f_{i-\frac{1}{2}}, & \text { for } i=1, \ldots, N / 4, \text { and } \\
\varepsilon \delta^{2} U_{i}+a_{i+\frac{1}{2}} D^{+} U_{i}-b_{i+\frac{1}{2}} U_{i+\frac{1}{2}}=f_{i+\frac{1}{2}}, & \text { for } i=3 N / 4, \ldots, N-1, \text { and } \\
& \text { when } \varepsilon \leq 2\|a\| N^{-1}, \\
D^{F} U_{i}-D^{B} U_{i}=0, \quad \text { for } i=N / 2, &
\end{array}\right.
$$

where

$$
\left\{\begin{array}{l}
D^{F} U_{N / 2}=\left(-U_{N / 2+2}+4 U_{N / 2+1}-3 U_{N / 2}\right) / 2 h_{(r)} \\
D^{B} U_{N / 2}=\left(U_{N / 2-2}-4 U_{N / 2-1}+3 U_{N / 2}\right) / 2 h_{(l)}
\end{array}\right.
$$


After rearranging the terms, we obtain the following form of the difference scheme on the mesh $\Omega^{N}$ :

$$
\left\{\begin{array}{l}
L_{\varepsilon}^{N} U_{i}=\tilde{f}_{i}, \quad \text { for } i=1, \ldots, N-1 \\
U_{0}=u_{0}, \quad U_{N}=u_{1}
\end{array}\right.
$$

where we define the finite difference operator $L_{\varepsilon}^{N}$ as

$$
L_{\varepsilon}^{N} U_{i}=\left\{\begin{array}{l}
{\left[r_{i}^{-} U_{i-1}+r_{i}^{0} U_{i}+r_{i}^{+} U_{i+1}\right], \quad i=1, \ldots, \frac{N}{2}-1, \frac{N}{2}+1, \ldots, N-1} \\
{\left[q_{i}^{-, 2} U_{i-2}+q_{i}^{-, 1} U_{i-1}+q_{i}^{0} U_{i}+q_{i}^{+, 1} U_{i+1}+q_{i}^{+, 2} U_{i+2}\right], \quad i=\frac{N}{2}}
\end{array}\right.
$$

and the right hand side vector $\tilde{f}_{i}$ as

$$
\widetilde{f}_{i} \equiv \begin{cases}f_{i}, & \text { for } i=N / 4+1, \ldots, N / 2-1, N / 2+1, \ldots, 3 N / 4-1, \\ f_{i}, & \text { for } i=1, \ldots, N / 4,3 N / 4, \ldots, N-1 \text { and } \varepsilon>2\|a\| N^{-1}, \\ f_{i-\frac{1}{2}}, & \text { for } i=1, \ldots, N / 4 \text { and when } \varepsilon \leq 2\|a\| N^{-1} \\ f_{i+\frac{1}{2}}, & \text { for } i=3 N / 4, \ldots, N-1 \text { and when } \varepsilon \leq 2\|a\| N^{-1} \\ 0, & \text { for } i=N / 2 .\end{cases}
$$

Let us denote

$$
\left\{\begin{array}{l}
\lambda=\varepsilon+\frac{1}{2} a_{i} h_{i}, \quad \text { for } i=1, \ldots, N / 2-1 \\
\mu=\varepsilon-\frac{1}{2} a_{i} h_{i+1}, \quad \text { for } i=N / 2+1, \ldots, N-1
\end{array}\right.
$$

Then, the coefficients in (3.2) for $i=N / 4+1, \ldots, N / 2-1$ and $i=N / 2+$ $1, \ldots, 3 N / 4-1$ are respectively given by

$$
\left\{\begin{array} { l } 
{ r _ { i } ^ { - } = \frac { 2 \lambda } { h _ { i + 1 } \widehat { h } _ { i } } , } \\
{ r _ { i } ^ { 0 } = - \frac { 2 \lambda } { h _ { i } h _ { i + 1 } } + \frac { a _ { i } } { h _ { i } } - b _ { i } , } \\
{ r _ { i } ^ { + } = \frac { 2 \lambda } { h _ { i } \widehat { h } _ { i } } - \frac { a _ { i } } { h _ { i } } , }
\end{array} \quad \left\{\begin{array}{l}
r_{i}^{-}=\frac{2 \mu}{h_{i} \widehat{h}_{i}}, \\
r_{i}^{0}=-\frac{2 \mu}{h_{i} h_{i+1}}-\frac{a_{i}}{h_{i+1}}-b_{i} \\
r_{i}^{+}=\frac{2 \mu}{h_{i+1} \widehat{h}_{i}}+\frac{a_{i}}{h_{i+1}}
\end{array}\right.\right.
$$

Next, when $\varepsilon>2\|a\| N^{-1}$ and $\varepsilon \leq 2\|a\| N^{-1}$, the coefficients in (3.2) for $i=$ $1, \ldots, N / 4$, are respectively given by

$$
\left\{\begin{array} { l } 
{ r _ { i } ^ { - } = \frac { 2 \lambda } { h _ { i + 1 } \widehat { h } _ { i } } , } \\
{ r _ { i } ^ { 0 } = - \frac { 2 \lambda } { h _ { i } h _ { i + 1 } } + \frac { a _ { i } } { h _ { i } } - b _ { i } , } \\
{ r _ { i } ^ { + } = \frac { 2 \lambda } { h _ { i } \widehat { h } _ { i } } - \frac { a _ { i } } { h _ { i } } , }
\end{array} \quad \left\{\begin{array}{rl}
r_{i}^{-} & =\frac{2 \varepsilon}{h_{i} \widehat{h}_{i}}-\frac{a_{i-1 / 2}}{h_{i}}-\frac{b_{i-1 / 2}}{2} \\
r_{i}^{0} & =-\frac{2 \varepsilon}{h_{i} h_{i+1}}+\frac{a_{i-1 / 2}}{h_{i}}-\frac{b_{i-1 / 2}}{2} \\
r_{i}^{+} & =\frac{2 \varepsilon}{h_{i+1} \widehat{h}_{i}} .
\end{array}\right.\right.
$$


Again, when $\varepsilon>2\|a\| N^{-1}$ and $\varepsilon \leq 2\|a\| N^{-1}$, the coefficients in (3.2) for $i=$ $3 N / 4, \ldots, N-1$ are respectively given by

$$
\left\{\begin{array} { l } 
{ r _ { i } ^ { - } = \frac { 2 \mu } { ( h _ { i } \widehat { h } _ { i } ) } , } \\
{ r _ { i } ^ { 0 } = - \frac { 2 \mu } { ( h _ { i } h _ { i + 1 } ) } - \frac { a _ { i } } { h _ { i + 1 } } - b _ { i } , } \\
{ r _ { i } ^ { + } = \frac { 2 \mu } { ( h _ { i + 1 } \widehat { h } _ { i } ) } + \frac { a _ { i } } { h _ { i + 1 } } , }
\end{array} \quad \left\{\begin{array}{rl}
r_{i}^{-} & =\frac{2 \varepsilon}{h_{i} \widehat{h}_{i}}, \\
r_{i}^{0} & =-\frac{2 \varepsilon}{\left(h_{i} h_{i+1}\right)}-\frac{a_{i+1 / 2}}{h_{i+1}}-\frac{b_{i+1 / 2}}{2} \\
r_{i}^{+} & =\frac{2 \varepsilon}{\left(h_{i+1} \widehat{h}_{i}\right)}+\frac{a_{i+1 / 2}}{h_{i+1}}-\frac{b_{i+1 / 2}}{2}
\end{array}\right.\right.
$$

Finally, the coefficients in (3.2) for $i=N / 2$ are given by

$$
\left\{\begin{array}{l}
q_{N / 2}^{-, 2}=-1 /\left(2 h_{(l)}\right), \quad q_{N / 2}^{-, 1}=2 / h_{(l)} \\
q_{N / 2}^{0}=-\frac{3}{2}\left(\frac{1}{h_{(l)}}+\frac{1}{h_{(r)}}\right) \\
q_{N / 2}^{+, 1}=2 / h_{(r)}, \quad q_{N / 2}^{+, 2}=-1 /\left(2 h_{(r)}\right)
\end{array}\right.
$$

\section{Stability}

In this section, we discuss the stability of the proposed numerical scheme (3.1)(3.3). In the analysis, we assume that $\sigma_{1}=\sigma_{2}=\sigma=\sigma_{0} \varepsilon \ln N$ and so,

$$
h_{i}= \begin{cases}H_{(l)}=4(\xi-\sigma) / N, & \text { for } i=1, \ldots, N / 4 \\ h=h_{(l)}=h_{(r)}=4 \sigma / N, & \text { for } i=N / 4+1, \ldots, 3 N / 4 \\ H_{(r)}=4(1-\xi-\sigma) / N, & \text { for } i=3 N / 4+1, \ldots, N\end{cases}
$$

It can be easily checked that the finite difference operator $L_{\varepsilon}^{N}$ defined in (3.2) does not satisfy the monotonicity property and accordingly, the present form (3.1) does not allow us to establish the stability result of the proposed numerical scheme. In order to overcome this difficulty, we transform the equation in (3.1) for $i=N / 2$, i.e.,

$$
q_{i}^{-, 2} U_{i-2}+q_{i}^{-, 1} U_{i-1}+q_{i}^{0} U_{i}+q_{i}^{+, 1} U_{i+1}+q_{i}^{+, 2} U_{i+2}=0
$$

so that the operator associated with the new system of equations satisfy the monotonicity property. Now, from (3.1) we have

$$
\left\{\begin{array}{l}
U_{N / 2-2}=\frac{1}{r_{N / 2-1}^{-}}\left[f_{N / 2-1}-r_{N / 2-1}^{0} U_{N / 2-1}-r_{N / 2-1}^{+} U_{N / 2}\right] \\
U_{N / 2+2}=\frac{1}{r_{N / 2+1}^{+}}\left[f_{N / 2+1}-r_{N / 2+1}^{0} U_{N / 2+1}-r_{N / 2+1}^{-} U_{N / 2}\right]
\end{array}\right.
$$

Therefore, after inserting the above expressions for $U_{N / 2-2}$ and $U_{N / 2+2}$ in (4.1), we transform the system of equations in (3.2) into a tridiagonal system of equations and obtain the following form:

$$
\left\{\begin{array}{l}
L_{h y b}^{N} U_{i}=\widetilde{f_{h y b}, i}, \quad \text { for } i=1, \ldots, N-1 \\
U_{0}=u_{0}, \quad U_{N}=u_{1}
\end{array}\right.
$$


Here, the difference operator $L_{h y b}^{N}$ and the right hand side term $\widetilde{f_{h y b}, i}$ are respectively defined as

$$
L_{h y b}^{N} U_{i}= \begin{cases}q_{i}^{-} U_{i-1}+q_{i}^{0} U_{i}+q_{i}^{+} U_{i+1}, & \text { for } i=N / 2, \\ L_{\varepsilon}^{N} U_{i}, & \text { for } i \neq N / 2\end{cases}
$$

and

$$
\widetilde{f_{h y b}, i}= \begin{cases}\frac{h / 2}{\lambda-h a_{i-1}} f_{i-1}+\frac{h / 2}{\mu+h a_{i+1}} f_{i+1}, & \text { for } i=N / 2, \\ \widetilde{f}_{i}, & \text { for } i \neq N / 2,\end{cases}
$$

where

$$
\left\{\begin{array}{l}
q_{N / 2}^{-}=\frac{1}{2 h}\left(4-\frac{2 \lambda-h a_{N / 2-1}+h^{2} b_{N / 2-1}}{\lambda-h a_{N / 2-1}}\right), \\
q_{N / 2}^{+}=\frac{1}{2 h}\left(4-\frac{2 \mu+h a_{N / 2+1}+h^{2} b_{N / 2+1}}{\mu+h a_{N / 2+1}}\right), \\
q_{N / 2}^{0}=\frac{1}{2 h}\left(-6+\frac{\lambda}{\lambda-h a_{N / 2-1}}+\frac{\mu}{\mu+h a_{N / 2+1}}\right) .
\end{array}\right.
$$

The following lemma shows that operator $L_{h y b}^{N}$ associated with the discrete problem (4.2) satisfies the monotonicity property with some mild assumptions.

Lemma 1. Assume that there exists a positive integer $N_{0}$ such that for all $N \geq N_{0}$,

$$
\frac{N}{\ln N} \geq 2 \sigma_{0} \alpha^{*}, \quad \frac{\alpha N}{2} \geq\|b\|
$$

where $\alpha=\min \left\{\alpha_{1}, \alpha_{2}\right\}$ and $\alpha^{*}=\max \left\{\alpha_{1}^{*}, \alpha_{2}^{*}\right\}$. Then, the operator $L_{h y b}^{N}$ defined by (4.3) satisfies a discrete maximum principle, i.e., if the mesh function $Z_{i}$ satisfies

$$
Z_{0} \leq 0, Z_{N} \leq 0 \quad \text { and } \quad L_{h y b}^{N} Z_{i} \geq 0 \quad \text { for } i=1, \ldots, N-1,
$$

then $Z_{i} \leq 0$ for all $i$.

Proof. Here, we set

$$
\widetilde{L_{h y b}^{N}} Z_{i}=-L_{h y b}^{N} Z_{i} \equiv A_{i, i-1} Z_{i-1}+A_{i, i} Z_{i}+A_{i, i+1} Z_{i+1}, \quad i=1, \ldots, N-1,
$$

where

$$
\begin{aligned}
& A_{i, i-1}=-r_{i}^{-}, \quad A_{i, i}=-r_{i}^{0}, \quad A_{i, i+1}=-r_{i}^{+}, \quad \text { for } i \neq N / 2 \\
& A_{N / 2, N / 2-1}=-\frac{1}{2 h}\left(\frac{2 \lambda-3 h a_{N / 2-1}-h^{2} b_{N / 2-1}}{\lambda-h a_{N / 2-1}}\right), \\
& A_{N / 2, N / 2+1}=-\frac{1}{2 h}\left(\frac{2 \mu+3 h a_{N / 2+1}-h^{2} b_{N / 2+1}}{\mu+h a_{N / 2+1}}\right), \\
& A_{N / 2, N / 2}=\frac{1}{2 h}\left(\frac{2 \lambda-3 h a_{N / 2-1}}{\lambda-h a_{N / 2-1}}+\frac{2 \mu+3 h a_{N / 2+1}}{\mu+h a_{N / 2+1}}\right) .
\end{aligned}
$$


Then, according to the hypothesis of the discrete maximum principle we suppose that

$$
\begin{aligned}
& \widetilde{L_{h y b}^{N}} Z_{i}=\rho_{i}, \quad \text { for } i=1, \ldots, N-1, \\
& Z_{0}=\rho_{0}, \quad Z_{N}=\rho_{N}, \quad \rho_{i} \leq 0, \text { for all } i .
\end{aligned}
$$

In this proof, we consider the following two cases based on the relation between $\varepsilon$ and $N$.

Case 1. When $\varepsilon>2\|a\| N^{-1}$. Here, we have

$$
\begin{aligned}
& \lambda \geq \varepsilon-\frac{\|a\|}{2 h_{i}}>0, \quad \text { for } 1 \leq i \leq N / 2-1 \\
& \mu \geq \varepsilon-\frac{\|a\|}{2 h_{i+1}}>0, \quad \text { for } N / 2+1 \leq i \leq N-1 .
\end{aligned}
$$

Now, let $1 \leq i \leq N / 2-1$ and $N / 2+1 \leq i \leq N-1$. Then, it is straightforward that

$$
A_{i, i-1}<0, A_{i, i+1}<0, \quad A_{i, i}>0, \quad\left|A_{i, i}\right|-\left|A_{i, i-1}\right|-\left|A_{i, i+1}\right| \geq 0 .
$$

Next, let $i=N / 2$. Then, using $h \leq 4 N^{-1}$ and the second assumption of (4.6), we have

$$
\begin{aligned}
& -3 a_{N / 2-1} / h-b_{N / 2-1}>3 \alpha_{1} N / 4-\|b\|>0, \\
& 3 a_{N / 2+1} / h-b_{N / 2+1}>3 \alpha_{2} N / 4-\|b\|>0,
\end{aligned}
$$

which imply that $A_{i, i-1}<0, A_{i, i+1}<0$. It is also clear that $A_{i, i}>0$ and $\left|A_{i, i}\right|-\left|A_{i, i}\right|-\left|A_{i, i+1}\right| \geq 0$.

Case 2. When $\varepsilon \leq 2\|a\| N^{-1}$. Here, using the first assumption of (4.6) we have

$$
\begin{aligned}
& \lambda=h\left(\frac{\varepsilon}{h}+\frac{a_{i}}{2}\right)=h\left(\frac{N}{4 \sigma_{0} \ln N}+\frac{a_{i}}{2}\right)>0, \quad \text { for } N / 4+1 \leq i \leq N / 2-1 \\
& \mu=h\left(\frac{\varepsilon}{h}-\frac{a_{i}}{2}\right)=h\left(\frac{N}{4 \sigma_{0} \ln N}-\frac{a_{i}}{2}\right)>0, \quad \text { for } N / 2+1 \leq i \leq 3 N / 4-1 .
\end{aligned}
$$

Now, for $N / 4+1 \leq i \leq 3 N / 4-1$, the similar arguments as in Case 1 can be used to show that

$$
A_{i, i-1}<0, \quad A_{i, i+1}<0, \quad A_{i, i}>0, \quad\left|A_{i, i}\right|-\left|A_{i, i-1}\right|-\left|A_{i, i+1}\right| \geq 0 .
$$

On the other hand, it is straightforward that $A_{i, i+1}<0, A_{i, i}>0$ for $1 \leq$ $i \leq N / 4$ and $A_{i, i-1}<0, A_{i, i}>0$ for $3 N / 4 \leq i \leq N-1$. Again, using $H_{(l)}, H_{(r)} \leq 4 N^{-1}$ and the second assumption of (4.6), we have

$$
\begin{aligned}
& -a_{i-\frac{1}{2}} / H_{(l)}-b_{i-\frac{1}{2}}>\alpha_{1} N / 4-\|b\| / 2>0 \\
& -a_{i+\frac{1}{2}} / H_{(r)}-b_{i+\frac{1}{2}}>\alpha_{2} N / 4-\|b\| / 2>0
\end{aligned}
$$


which imply that $A_{i, i-1}<0$ for $1 \leq i \leq N / 4$ and $A_{i, i+1}>0$ for $3 N / 4 \leq i \leq$ $N-1$. Moreover, for both $1 \leq i \leq N / 4$ and $3 N / 4 \leq i \leq N-1$, it is clear that $\left|A_{i, i}\right|-\left|A_{i, i-1}\right|-\left|A_{i, i+1}\right| \geq 0$.

Therefore, in both the cases under the assumptions (4.6), it can be shown that the $(N+1) \times(N+1)$ matrix formed by the coefficients of the mesh function $Z_{i}$ in (4.7) for $i=0, \ldots, N$ is an $M$-matrix and therefore, the result follows.

Now, as an immediate consequence of the above discrete maximum principle, one can deduce the $\varepsilon$-uniform stability of the scheme (4.2)-(4.5) stated in the following lemma.

Lemma 2. Let $U$ be the solution of (4.2)-(4.5) and let the assumptions (4.6) of Lemma 1 hold true. Then

$$
\|U\|_{\bar{\Omega}^{N}} \leq \max \left\{\left|u_{0}\right|,\left|u_{1}\right|\right\}+\frac{1}{\eta}\left\|\widetilde{f_{h y b}}\right\|_{\bar{\Omega}^{N}},
$$

where $\eta=\min \left\{\alpha_{1} / \xi, \alpha_{2} /(1-\xi)\right\}$.

\section{Error Analysis}

In this section, we establish the $\varepsilon$-uniform error estimate of the hybrid scheme (4.2)-(4.5). Here, we decompose the discrete solution $U$ into the smooth and the non-smooth components as

$$
U_{i}= \begin{cases}V_{L, i}+W_{L, i}, & \text { for } i=1, \ldots, N / 2-1 \\ V_{L, i}+W_{L, i}=V_{R, i}+W_{R, i}, & \text { for } i=N / 2 \\ V_{R, i}+W_{R, i}, & \text { for } i=N / 2+1, \ldots, N-1,\end{cases}
$$

where the smooth components $V_{L}$ and $V_{R}$ (which approximate $v$ respectively to the left and to the right of the point of discontinuity $x=\xi$ ) are respectively defined as the solutions of the discrete problems

$$
\left\{\begin{array}{l}
L_{h y b}^{N} V_{L, i}={\widetilde{f_{h y b}, i}}^{n+1}, \quad \text { for } i=1, \ldots, N / 2-1 \\
V_{L, 0}=v(0), \quad V_{L, N / 2}=v\left(\xi^{-}\right)
\end{array}\right.
$$

and

$$
\left\{\begin{array}{l}
L_{h y b}^{N} V_{R, i}=\widetilde{f_{h y b}, i}, \quad \text { for } i=N / 2+1, \ldots, N-1, \\
V_{R, N / 2}=v\left(\xi^{+}\right), \quad V_{R, N}=v(1) .
\end{array}\right.
$$

Hence, the non-smooth components $W_{L}$ and $W_{R}$ (which approximate $w$ on either side of $x=\xi$ ) must satisfy the following system of equations

$$
\left\{\begin{array}{l}
L_{h y b}^{N} W_{L, i}=0, \quad \text { for } i=1, \ldots, N / 2-1 \\
L_{h y b}^{N} W_{R, i}=0, \quad \text { for } i=N / 2+1, \ldots, N-1 \\
W_{L, 0}=0, \quad W_{R, N}=0 \\
W_{R, N / 2}+V_{R, N / 2}=W_{L, N / 2}+V_{L, N / 2} \\
D^{F} W_{R, N / 2}+D^{F} V_{R, N / 2}=D^{B} W_{L, N / 2}+D^{B} V_{L, N / 2}
\end{array}\right.
$$

In the following sections, we analyze the errors separately in the outer region (i.e., for $\left.x_{i} \in \bar{\Omega}^{N} \bigcap[(0, \xi-\sigma] \cup[\xi+\sigma, 1)]\right)$ and in the interior layer region (i.e., for $\left.x_{i} \in \bar{\Omega}^{N} \cap(\xi-\sigma, \xi+\sigma)\right)$. 


\subsection{Error in the outer region}

In the following lemma, we obtain the error bounds associated with the smooth components.

Lemma 3. Under the assumptions (4.6) of Lemma 1, the errors associated with the smooth components satisfy the following estimates

$$
\left\{\begin{array}{l}
\left|V_{L, i}-v\left(x_{i}\right)\right| \leq C N^{-2}, \quad \text { for } 1 \leq i \leq N / 2-1 \\
\left|V_{R, i}-v\left(x_{i}\right)\right| \leq C N^{-2}, \quad \text { for } N / 2+1 \leq i \leq N-1
\end{array}\right.
$$

Proof. In this proof, we consider following two cases depending on the relation between $\varepsilon$ and $N$.

Case 1. When $\varepsilon>2\|a\| N^{-1}$. Let $1 \leq i \leq N / 2-1$. Here, we define the truncation error as

$$
L_{h y b}^{N}\left(V_{L, i}-v\left(x_{i}\right)\right)=\left(L_{\varepsilon}-L_{\varepsilon}^{N}\right) v\left(x_{i}\right)=\varepsilon\left(\frac{d^{2}}{d x^{2}}-\delta^{2}\right) v\left(x_{i}\right)+a_{i}\left(\frac{d}{d x}-D^{\star}\right) v\left(x_{i}\right) .
$$

Then, the truncation error satisfies the following estimate

$$
\left|L_{h y b}^{N}\left(V_{L, i}-v\left(x_{i}\right)\right)\right| \leq \begin{cases}C\left[\varepsilon \widehat{h}_{i}\left\|v^{(3)}\right\|+h_{i} h_{i+1}\left\|v^{(3)}\right\|\right], & \text { for } i=N / 4 \\ C\left[\varepsilon h_{i}^{2}\left\|v^{(4)}\right\|+h_{i}^{2}\left\|v^{(3)}\right\|\right], & \text { otherwise. }\end{cases}
$$

Now, using $h_{i} \leq C N^{-1}$ and the bounds of the derivatives of $v$ given in Theorem 1 , we obtain the following estimate

$$
\left|L_{h y b}^{N}\left(V_{L, i}-v\left(x_{i}\right)\right)\right| \leq \begin{cases}C\left[\varepsilon N^{-1}+N^{-2}\right], & \text { for } i=N / 4 \\ C N^{-2}, & \text { otherwise }\end{cases}
$$

Afterwards, by choosing the following barrier function

$$
\Psi_{L, i}=-C N^{-2} \theta_{L}\left(x_{i}\right)-C N^{-2} x_{i}, \quad \text { for } 0 \leq i \leq N / 2,
$$

where

$$
\theta_{L}(z)= \begin{cases}1, & \text { for } \xi-\sigma \leq z \leq \xi \\ z /(\xi-\sigma), & \text { for } 0 \leq z \leq \xi-\sigma\end{cases}
$$

and applying the discrete maximum principle (Lemma 1) to $\Psi_{L, i} \pm\left(V_{L, i}-v\left(x_{i}\right)\right)$ over $\bar{\Omega}^{N} \bigcap[0, \xi]$, yields the following estimate

$$
\left|V_{L, i}-v\left(x_{i}\right)\right| \leq-\Psi_{L, i} \leq C N^{-2}, \quad \text { for } 1 \leq i \leq N / 2-1 .
$$

Next, let $N / 2+1 \leq i \leq N-1$. Then, using the following barrier function

$$
\Psi_{R, i}=-C N^{-2} \theta_{R}\left(x_{i}\right)-C N^{-2}\left(1-x_{i}\right), \quad \text { for } N / 2 \leq i \leq N,
$$

where

$$
\theta_{R}(z)= \begin{cases}1, & \text { for } \xi \leq z \leq \xi+\sigma \\ \frac{1-z}{1-(\xi+\sigma)}, & \text { for } \xi+\sigma \leq z \leq 1\end{cases}
$$


and arguing similarly for $\left(V_{R}-v\right)$ on the right side of the discontinuity, one can obtain that

$$
\left|V_{R, i}-v\left(x_{i}\right)\right| \leq C N^{-2}, \quad \text { for } N / 2+1 \leq i \leq N-1
$$

Case 2. When $\varepsilon \leq 2\|a\| N^{-1}$. Here, the truncation errors for $1 \leq i \leq N / 2-1$ and $N / 2+1 \leq i \leq N-1$ are respectively defined as

$$
\begin{aligned}
& L_{h y b}^{N}\left(V_{L, i}-v\left(x_{i}\right)\right)=\left\{\begin{array}{l}
\left(L_{\varepsilon}-L_{\varepsilon}^{N}\right) v\left(x_{i}\right), \quad \text { for } N / 4+1 \leq i \leq N / 2-1, \\
\left(\left(L_{\varepsilon} v\right)_{i-1 / 2}-L_{\varepsilon}^{N} v\left(x_{i}\right)\right), \quad \text { for } 1 \leq i \leq N / 4,
\end{array}\right. \\
& L_{h y b}^{N}\left(V_{R, i}-v\left(x_{i}\right)\right)=\left\{\begin{array}{l}
\left(L_{\varepsilon}-L_{\varepsilon}^{N}\right) v\left(x_{i}\right), \quad \text { for } N / 2+1 \leq i \leq 3 N / 4-1, \\
\left(\left(L_{\varepsilon} v\right)_{i+1 / 2}-L_{\varepsilon}^{N} v\left(x_{i}\right)\right), \quad \text { for } 3 N / 4 \leq i \leq N-1 .
\end{array}\right.
\end{aligned}
$$

Now, using the assumption $\varepsilon \leq 2\|a\| N^{-1}$ and invoking Theorem 1 and Lemma 1 , by means of the barrier function approach as given in [1], one can obtain the desired error estimate. Hence, this completes the proof.

In the following lemma, we obtain the error bounds associated with the non-smooth components.

Lemma 4. Let $\gamma=\alpha / 2$. Then, under the assumptions (4.6) of Lemma 1, the errors associated to the non-smooth components satisfy the following estimates

$$
\left\{\begin{array}{l}
\left|W_{L, i}-w\left(x_{i}\right)\right| \leq C N^{-2}, \quad \text { for } 1 \leq i \leq N / 4 \\
\left|W_{R, i}-w\left(x_{i}\right)\right| \leq C N^{-2}, \quad \text { for } 3 N / 4 \leq i \leq N-1
\end{array}\right.
$$

Proof. To obtain the bound for $W_{L}$, we consider the function $\Phi_{L}$ as the solution of the following discrete problem

$$
\left\{\begin{array}{l}
\left(\varepsilon \delta^{2}-\gamma D^{-}\right) \Phi_{L, i}=0, \quad \text { for } i=1, \ldots, N / 2-1 \\
\Phi_{L, 0}=0, \quad \Phi_{L, N / 2}=1
\end{array}\right.
$$

satisfying the properties

$$
\Phi_{L, i} \geq 0, \text { for all } i, \quad D^{-} \Phi_{L, i} \geq 0, \text { for } 1 \leq i \leq N / 2-1
$$

and following the approach given in [ [3], Chapter 3], one can further deduce that there exists some constant $C$ such that $\Phi_{L, N / 4} \leq C N^{-2}$. We also have

$$
L_{h y b}^{N} \Phi_{L, i} \leq 0, \quad \text { for } 1 \leq i \leq N / 2-1
$$

since,

$$
\left[\varepsilon \delta^{2} \Phi_{L, i}+a_{i} D^{\star} \Phi_{L, i}-b_{i} \Phi_{L, i}\right]=\left[\frac{a_{i}}{2} h_{i} \delta^{2} \Phi_{L, i}+\left(\gamma+a_{i}\right) D^{-} \Phi_{L, i}-b_{i} \Phi_{L, i}\right] \leq 0,
$$

when $\varepsilon>2\|a\| N^{-1}$, and

$$
\left[\varepsilon \delta^{2} \Phi_{L, i}+a_{i-\frac{1}{2}} D^{-} \Phi_{L, i}-b_{i-\frac{1}{2}} \Phi_{L, i-\frac{1}{2}}\right]=\left[\left(\gamma+a_{i}\right) D^{-} \Phi_{L, i}-b_{i-\frac{1}{2}} \Phi_{L, i-\frac{1}{2}}\right] \leq 0
$$


when $\varepsilon \leq 2\|a\| N^{-1}$. Therefore, applying the discrete maximum principle (Lemma 1) to $-\left|W_{L, N / 2}\right| \Phi_{L, i} \pm W_{L, i}$, over $\bar{\Omega}^{N} \bigcap[0, \xi]$, we have

$$
\left|W_{L, i}\right| \leq\left|W_{L, N / 2}\right| \Phi_{L, i} \leq C \Phi_{L, N / 4} \leq C N^{-2}, \quad \text { for } 1 \leq i \leq N / 4 \text {. }
$$

Using Theorem 1 , we thus obtain for $1 \leq i \leq N / 4$ that

$$
\left|W_{L, i}-w\left(x_{i}\right)\right| \leq\left|W_{L, i}\right|+\left|w\left(x_{i}\right)\right| \leq C N^{-2}+C \exp (-\gamma \sigma / \varepsilon) \leq C N^{-2} .
$$

On the other hand, for $3 N / 4 \leq i \leq N-1$, by considering the function $\Phi_{R}$ as the solution of the following discrete problem

$$
\left\{\begin{array}{l}
\left(\varepsilon \delta^{2}+\gamma D^{+}\right) \Phi_{R, i}=0, \quad \text { for } i=N / 2+1, \ldots, N-1 \\
\Phi_{R, N / 2}=1, \quad \Phi_{R, N}=0
\end{array}\right.
$$

and arguing similarly for $\left(W_{R}-w\right)$ on the right side of the discontinuity, one can obtain that

$$
\left|W_{R, i}-w\left(x_{i}\right)\right| \leq C N^{-2}, \quad \text { for } 3 N / 4 \leq i \leq N-1 .
$$

Hence, this completes the proof.

Thus, in the outer region, the bound for $\left|U_{i}-u\left(x_{i}\right)\right|$ in the following lemma can be easily obtained from Lemmas 3 and 4 , by decomposing the error $(U-u)$ into the smooth and the non-smooth components as

$$
\begin{aligned}
& \left(U_{i}-u\left(x_{i}\right)\right)=\left(V_{L, i}-v\left(x_{i}\right)\right)+\left(W_{L, i}-w\left(x_{i}\right)\right), \quad \text { for } 1 \leq i \leq N / 4, \\
& \left(U_{i}-u\left(x_{i}\right)\right)=\left(V_{R, i}-v\left(x_{i}\right)\right)+\left(W_{R, i}-w\left(x_{i}\right)\right), \quad \text { for } 3 N / 4 \leq i \leq N-1 .
\end{aligned}
$$

Lemma 5. Let $\gamma=\alpha / 2$. Then, under the assumptions (4.6) of Lemma 1, the error associated with the hybrid scheme (4.2)-(4.5), satisfies that

$$
\left|U_{i}-u\left(x_{i}\right)\right| \leq C N^{-2}, \quad \text { for } 1 \leq i \leq N / 4 \text { and } 3 N / 4 \leq i \leq N-1 .
$$

\subsection{Error in the interior layer region}

On $\bar{\Omega}^{N}=\left\{x_{i}\right\}_{0}^{N}$, we define the following two mesh functions

$$
\begin{aligned}
& S_{i}=\prod_{j=1}^{i}\left(1+\frac{\gamma h_{j}}{\varepsilon}\right), \quad \text { for } 1 \leq i \leq N / 2, \\
& Q_{i}=\prod_{j=i+1}^{N}\left(1+\frac{\gamma h_{j}}{\varepsilon}\right), \quad \text { for } N / 2 \leq i \leq N-1
\end{aligned}
$$

(with the usual convention that $S_{0}=1$ and $Q_{N}=1$ ) where $\gamma$ is a positive constant. 
Lemma 6. On $\bar{\Omega}^{N}=\left\{x_{i}\right\}_{0}^{N}$, the mesh functions $S_{i}$ and $Q_{i}$ satisfy the following inequalities:

$$
\left\{\begin{array}{l}
\exp \left(-\gamma\left(\xi-x_{i}\right) / \varepsilon\right) \leq\left(S_{i} / S_{N / 2}\right), \quad \text { for } 1 \leq i \leq N / 2-1 \\
\exp \left(-\gamma\left(x_{i}-\xi\right) / \varepsilon\right) \leq\left(Q_{i} / Q_{N / 2}\right), \quad \text { for } N / 2+1 \leq i \leq N-1
\end{array}\right.
$$

Proof. The proof follows from [ [8], Lemma 5.7].

Lemma 7. Let $\gamma=\alpha / 2$. Then, for some constant $C$, the mesh functions $S_{i}$ and $Q_{i}$ satisfy that

$$
\left\{\begin{array}{l}
-L_{h y b}^{N} S_{i} \geq \frac{C}{\varepsilon+\gamma h} S_{i}, \quad \text { for } N / 4+1 \leq i \leq N / 2-1, \\
-L_{h y b}^{N} Q_{i} \geq \frac{C}{\varepsilon+\gamma h} Q_{i}, \quad \text { for } N / 2+1 \leq i \leq 3 N / 4-1 .
\end{array}\right.
$$

Proof. A straightforward calculation yields that

$$
\begin{aligned}
-L_{h y b}^{N} S_{i} & \geq-\frac{\gamma}{\varepsilon} S_{i-1}\left(\gamma+a_{i}\right)-a_{i} \frac{\gamma^{2} h}{2 \varepsilon^{2}} S_{i-1}, \\
& \geq \frac{C}{\varepsilon+\gamma h} S_{i}, \quad \text { for } N / 4+1 \leq i \leq N / 2-1, \\
-L_{h y b}^{N} Q_{i} & \geq \frac{\gamma}{\varepsilon} Q_{i+1}\left(a_{i}-\gamma\right)+a_{i} \frac{\gamma^{2} h}{2 \varepsilon^{2}} Q_{i+1}, \\
& \geq \frac{C}{\varepsilon+\gamma h} Q_{i}, \quad \text { for } N / 2+1 \leq i \leq 3 N / 4-1 .
\end{aligned}
$$

Hence the proof.

In the following lemma, the above technical lemmas are used to obtain the bound for $\left|U_{i}-u\left(x_{i}\right)\right|$ in the interior layer region.

Lemma 8. Let $\gamma=\alpha / 2$. Then, under the assumptions (4.6) of Lemma 1, the error associated with the hybrid scheme (4.2)-(4.5) satisfies that

$$
\left|U_{i}-u\left(x_{i}\right)\right| \leq C\left(N^{-2} \ln ^{2} N\right), \quad \text { for } N / 4+1 \leq i \leq 3 N / 4-1 .
$$

Proof. Here, for $N / 4+1 \leq i \leq N / 2-1$ and $N / 2+1 \leq i \leq 3 N / 4-1$, the truncation error is defined as

$$
\begin{aligned}
L_{h y b}^{N}\left(V_{L, i}-v\left(x_{i}\right)\right)=\left(L_{\varepsilon}-L_{\varepsilon}^{N}\right) v\left(x_{i}\right)= & \varepsilon\left(\frac{d^{2}}{d x^{2}}-\delta^{2}\right) v\left(x_{i}\right) \\
& +a_{i}\left(\frac{d}{d x}-D^{\star}\right) v\left(x_{i}\right) .
\end{aligned}
$$

Then, following the arguments given in [1], one can deduce that the truncation error satisfies the following estimates

$$
\begin{aligned}
& \left|L_{h y b}^{N}\left(U_{i}-u\left(x_{i}\right)\right)\right| \leq C\left(h^{2}+\frac{h^{2}}{\varepsilon^{3}} \exp \left(-\left(\xi-x_{i}\right) \alpha_{1} / \varepsilon\right)\right), \\
& \left|L_{h y b}^{N, M}\left(U_{i}-u\left(x_{i}\right)\right)\right| \leq C\left(h^{2}+\frac{h^{2}}{\varepsilon^{3}} \exp \left(-\left(x_{i}-\xi\right) \alpha_{2} / \varepsilon\right)\right)
\end{aligned}
$$


for $N / 4+1 \leq i \leq N / 2-1$ and $N / 2+1 \leq i \leq 3 N / 4-1$, respectively. Next, at the mesh point $x_{N / 2}=\xi$, we define the truncation error as

$$
\begin{aligned}
L_{h y b}^{N}\left(U_{N / 2}-u\left(x_{N / 2}\right)\right)= & \frac{h / 2}{\lambda-h a_{N / 2-1}} f_{N / 2-1}+\frac{h / 2}{\mu+h a_{N / 2+1}} f_{N / 2+1} \\
& -L_{h y b}^{N} u\left(x_{N / 2}\right)
\end{aligned}
$$

and also, we have the following estimate

$$
\begin{aligned}
\left|L_{h y b}^{N}\left(U_{N / 2}-u\left(x_{N / 2}\right)\right)\right| \leq & \frac{h / 2}{\lambda-h a_{N / 2-1}}\left|L_{h y b}^{N}\left(U_{N / 2-1}-u\left(x_{N / 2-1}\right)\right)\right| \\
& +\frac{h / 2}{\mu+h a_{N / 2+1}}\left|L_{h y b}^{N}\left(U_{N / 2+1}-u\left(x_{N / 2+1}\right)\right)\right| \\
& +\left|\left(D^{F}-D^{B}\right) u\left(x_{N / 2}\right)-\left[\frac{d u}{d x}\right]\left(x_{N / 2}\right)\right| \leq C \frac{h^{2}}{\varepsilon^{3}} .
\end{aligned}
$$

Again, from Lemma 5, we have

$$
\left|U_{i}-u\left(x_{i}\right)\right| \leq C N^{-2}, \quad \text { for } i=N / 4,3 N / 4 .
$$

We now define the following discrete function

$$
\varphi_{i}=\left\{\begin{array}{r}
-C N^{-2}\left(1+\left[x_{i}-(\xi-\sigma)\right]\right)-C\left(N^{-2} \ln ^{2} N\right)\left(S_{i} / S_{N / 2}\right), \\
\text { for } N / 4 \leq i \leq N / 2, \\
-C N^{-2}\left(1+\left[(\xi+\sigma)-x_{i}\right]\right)-C\left(N^{-2} \ln ^{2} N\right)\left(Q_{i} / Q_{N / 2}\right), \\
\text { for } N / 2 \leq i \leq 3 N / 4,
\end{array}\right.
$$

where $C$ is chosen sufficiently large. Then, a straightforward calculation using Lemmas 6 and 7 together with the assumptions (4.6), implies that

$$
L_{h y b}^{N} \varphi_{i} \geq\left\{\begin{array}{r}
C \alpha_{1} N^{-2}+C\left(\varepsilon^{-1} N^{-2} \ln ^{2} N\right) \exp \left(-\left(\xi-x_{i}\right) \alpha_{1} / \varepsilon\right), \\
\text { for } N / 4+1 \leq i \leq N / 2-1 \\
C \alpha_{2} N^{-2}+C\left(\varepsilon^{-1} N^{-2} \ln ^{2} N\right) \exp \left(-\left(x_{i}-\xi\right) \alpha_{2} / \varepsilon\right), \\
\text { for } N / 2+1 \leq i \leq 3 N / 4-1
\end{array}\right.
$$

and

$$
L_{h y b}^{N} \varphi_{N / 2} \geq 2 C N^{-2}+2 C\left(\varepsilon^{-1} N^{-2} \ln ^{2} N\right) \geq\left|L_{h y b}^{N}\left(U_{N / 2}-u\left(x_{N / 2}\right)\right)\right| .
$$

Therefore, applying the discrete maximum principle to $\varphi_{i} \pm\left(U_{i}^{n}-u\left(x_{i}, t_{n}\right)\right)$ over the domain $\bar{\Omega}^{N} \cap[\xi-\sigma, \xi+\sigma]$, we obtain the desired result. Hence, the proof.

\subsection{The main convergence result}

Theorem 2. Assume that $N \geq N_{0}$, satisfies the conditions given in (4.6). Then, if $\gamma=\alpha / 2$, the error associated with the hybrid scheme (4.2)-(4.5) satisfies that

$$
\left|U_{i}-u\left(x_{i}\right)\right| \leq\left\{\begin{array}{l}
C N^{-2}, \quad \text { for } 1 \leq i \leq N / 4 \text { and } 3 N / 4 \leq i<N-1 \\
C N^{-2} \ln ^{2} N, \quad \text { for } \quad N / 4+1 \leq i \leq 3 N / 4-1
\end{array}\right.
$$


Proof. The proof follows from Lemma 5 and Lemma 8.

Remark 1. It is to be noted that the the hybrid scheme given in [1] satisfies the above error estimate under the restrictive assumption that the perturbation parameter $\varepsilon<C N^{-1}$. In fact, the numerical experiments performed in Section 6 also demonstrate that the scheme given in [1] is first-order uniformly convergent with respect to $\varepsilon$ in the discrete supremum norm.

\section{$6 \quad$ Numerical Experiments}

In this section, we present the numerical results obtained by the newly proposed scheme (3.1)-(3.3) and also compare the numerical results obtained by the proposed scheme with the hybrid scheme-II. To do this, we conduct the numerical experiments for various types of test examples which includes the BVPs of the form (1.1)-(1.2).

\subsection{Hybrid Scheme-II}

Here, we describe the hybrid scheme proposed by Cen in [1] for the BVP of the form (1.1)-(1.2) and the numerical scheme takes the following form:

$$
\begin{cases}\varepsilon \delta^{2} U_{i}+a_{i} D^{0} U_{i}-b_{i} U_{i}=f_{i}, \quad \text { for } i=N / 4+1, \ldots, N / 2-1, N / 2+1, \ldots, & 3 N / 4-1, \\ \varepsilon \delta^{2} U_{i}+a_{i-\frac{1}{2}} D^{-} U_{i}-b_{i-\frac{1}{2}} U_{i-\frac{1}{2}}=f_{i-\frac{1}{2}}, & \text { for } 1, \ldots, N / 4, \\ \varepsilon \delta^{2} U_{i}+a_{i+\frac{1}{2}} D^{+} U_{i}-b_{i+\frac{1}{2}} U_{i+\frac{1}{2}}=f_{i+\frac{1}{2}}, & \text { for } 3 N / 4, \ldots, N-1, \\ \frac{-U_{i+2}+4 U_{i+1}-3 U_{i}}{2 h_{(r)}}-\frac{U_{i-2}-4 U_{i-1}+3 U_{i}}{2 h_{(l)}}=0, \quad \text { for } i=N / 2, & \\ U_{0}=u_{0}, \quad U_{N}=u_{1}, & \end{cases}
$$

where, for a given mesh function $Z\left(x_{i}\right)=Z_{i}, Z_{i \pm \frac{1}{2}}$, the finite difference operators $\delta^{2}, D^{-}, D^{+}$are defined in Section 3 and the central difference operator $D^{0}$ is defined by

$$
D^{0} Z_{i}=\left(Z_{i+1}-Z_{i-1}\right) / \hat{h}_{i}
$$

\subsection{Test example in which exact solution is known}

Example 1. Consider the following BVP of the form (1.1)-(1.2):

$$
\left\{\begin{array}{l}
\varepsilon u^{\prime \prime}(x)-(1+x(0.5-x)) u^{\prime}(x)-(1+x) u(x)=f(x), \quad x \in(0,0.5), \\
\varepsilon u^{\prime \prime}(x)+(1+x(x-0.5)) u^{\prime}(x)-(1+x) u(x)=f(x), \quad x \in(0.5,1), \\
{[u](0.5)=0, \quad\left[\frac{d u}{d x}\right](0.5)=0,} \\
u(0)=0, \quad u(1)=0,
\end{array}\right.
$$

where the source function $f(x)$ has been chosen to fit

$$
u(x)=\frac{1-\exp (-(0.5-x) / \varepsilon)}{1-\exp (-0.5 / \varepsilon)}-\cos (\pi x),
$$


as the exact solutions of the above problem. In Figure 1, we display the exact solution $u$ of the BVP (6.1) which clearly shows that the gradient of the solution $u$ steepens near the point of discontinuity $x=0.5$ as the parameter $\varepsilon$ decreases.

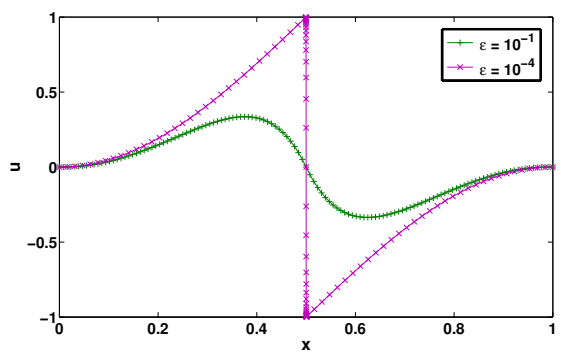

Figure 1. Exact solution for Example 1 on the mesh $\bar{\Omega}^{N}$ for $N=128$.

For each $\varepsilon$, we calculate the maximum point-wise error by

$$
e_{\varepsilon}^{N}=\max _{x_{i} \in \bar{\Omega}^{N}}\left|u\left(x_{i}\right)-U_{i}^{N}\right|,
$$

where $u$ and $U^{N}$ denote the exact and the numerical solution obtained on the mesh $\bar{\Omega}^{N}$. In addition to this, we determine the corresponding order of convergence by

$$
p_{\varepsilon}^{N}=\log _{2}\left(e_{\varepsilon}^{N} / e_{\varepsilon}^{2 N}\right) .
$$

Now, for each $N$, we define $e^{N}=\max _{\varepsilon} e_{\varepsilon}^{N}$ as the $\varepsilon$-uniform maximum point-wise error and the corresponding $\varepsilon$-uniform order of convergence is defined by

$$
p^{N}=\log _{2}\left(e^{N} / e^{2 N}\right) .
$$

For various values of $\varepsilon$ and $N$, the maximum point-wise errors $e_{\varepsilon}^{N}$ and the corresponding order of convergence $p_{\varepsilon}^{N}$ computed using the newly proposed scheme (3.1)-(3.3) and the hybrid scheme-II for Example 1 are presented in Table 1. We also display the $\varepsilon$-uniform errors $e^{N}$ and the corresponding order of convergence $p^{N}$ computed using both the hybrid schemes in Table 1 .

In spite of this, for clarity of the presentation, the errors associated with the numerical solutions of the BVP (6.1) computed using both the hybrid schemes for $\varepsilon=10^{-1}$ and the corresponding zoomed view of the errors near $x=0.5$ for $\varepsilon=10^{-4}$ are depicted in Figure 2 .

\subsection{Test examples in which exact solutions are not known}

Example 2. Consider the following BVP of the form (1.1)-(1.2):

$$
\left\{\begin{array}{l}
\varepsilon u^{\prime \prime}(x)-u^{\prime}(x)=0.5, \quad x \in(0,0.5) \\
\varepsilon u^{\prime \prime}(x)+u^{\prime}(x)=1, \quad x \in(0.5,1) \\
{[u](0.5)=0, \quad[d u / d x](0.5)=0} \\
u(0)=0, \quad u(1)=1
\end{array}\right.
$$


Table 1. Maximum point-wise errors and order of convergence for Example 1.

\begin{tabular}{|c|c|c|c|c|c|c|c|c|}
\hline \multirow[t]{3}{*}{$\varepsilon$} & \multicolumn{8}{|c|}{ Number of mesh intervals, $N$} \\
\hline & \multicolumn{2}{|c|}{32} & \multicolumn{2}{|r|}{64} & \multicolumn{2}{|c|}{128} & \multicolumn{2}{|c|}{256} \\
\hline & $\begin{array}{l}\text { new } \\
\text { scheme }\end{array}$ & $\begin{array}{l}\text { scheme } \\
\text { II }\end{array}$ & $\begin{array}{l}\text { new } \\
\text { scheme }\end{array}$ & $\begin{array}{l}\text { scheme } \\
\text { II }\end{array}$ & $\begin{array}{l}\text { new } \\
\text { scheme }\end{array}$ & $\begin{array}{l}\text { scheme } \\
\text { II }\end{array}$ & $\begin{array}{l}\text { new } \\
\text { scheme }\end{array}$ & $\begin{array}{l}\text { scheme } \\
\text { II }\end{array}$ \\
\hline \multirow[t]{2}{*}{1} & $6.951 \mathrm{e}-05$ & $4.780 \mathrm{e}-03$ & $1.739 \mathrm{e}-$ & 2.3 & $4.354 \mathrm{e}-06$ & $1.141 \mathrm{e}$ & $1.088 \mathrm{e}-06$ & 5.6 \\
\hline & 1.998 & 1.045 & 1.997 & 1.020 & 2.000 & 1.011 & 2.000 & 1.005 \\
\hline \multirow[t]{2}{*}{$10^{-1}$} & $3.209 \mathrm{e}-03$ & $9.582 \mathrm{e}-03$ & $7.955 \mathrm{e}-04$ & $4.822 \mathrm{e}-03$ & $1.984 \mathrm{e}-04$ & $2.434 \mathrm{e}-03$ & $4.962 \mathrm{e}-05$ & $1.221 \mathrm{e}-03$ \\
\hline & 2.012 & 0.990 & 2.003 & 0.986 & 1.999 & 0.995 & 2.000 & 0.997 \\
\hline \multirow[t]{2}{*}{$10^{-2}$} & $2.314 \mathrm{e}-02$ & $2.314 \mathrm{e}-02$ & $7.825 \mathrm{e}-03$ & $7.825 \mathrm{e}-03$ & $2.594 \mathrm{e}-03$ & $2.594 \mathrm{e}-03$ & $8.179 \mathrm{e}-04$ & $8.179 \mathrm{e}-04$ \\
\hline & 1.564 & 1.564 & 1.593 & 1.593 & 1.665 & 1.665 & 1.488 & 1.703 \\
\hline \multirow[t]{2}{*}{$10^{-3}$} & $2.364 \mathrm{e}-02$ & $2.364 \mathrm{e}-02$ & $8.123 \mathrm{e}-03$ & $8.123 \mathrm{e}-03$ & $2.741 \mathrm{e}-03$ & $2.741 \mathrm{e}-03$ & $8.881 \mathrm{e}-04$ & $8.881 \mathrm{e}-04$ \\
\hline & 1.541 & 1.541 & 1.567 & 1.567 & 1.626 & 1.626 & 1.668 & 1.668 \\
\hline \multirow[t]{2}{*}{$10^{-4}$} & $2.371 \mathrm{e}-02$ & $2.371 \mathrm{e}-02$ & $8.171 \mathrm{e}-03$ & $8.1717 \mathrm{e}-03$ & $2.767 \mathrm{e}-03$ & $2.767 \mathrm{e}-03$ & $9.023 \mathrm{e}-04$ & $9.023 \mathrm{e}-04$ \\
\hline & 1.537 & 1.537 & 1.562 & 1.562 & 1.616 & 1.616 & 1.655 & 1.654 \\
\hline \multirow[t]{2}{*}{$10^{-5}$} & $2.372 \mathrm{e}-02$ & $2.372 \mathrm{e}-02$ & $8.176 \mathrm{e}-03$ & $8.176 \mathrm{e}-03$ & $2.769 \mathrm{e}-03$ & $2.769 \mathrm{e}-03$ & $9.038 \mathrm{e}-04$ & $9.038 \mathrm{e}-04$ \\
\hline & 1.536 & 1.536 & 1.561 & 1.561 & 1.615 & 1.615 & 1.653 & 1.653 \\
\hline \multirow[t]{2}{*}{$10^{-6}$} & $2.372 \mathrm{e}-02$ & $2.372 \mathrm{e}-02$ & $8.177 \mathrm{e}-03$ & $8.177 \mathrm{e}-03$ & $2.770 \mathrm{e}-03$ & $2.770 \mathrm{e}-03$ & $9.040 \mathrm{e}-04$ & $9.040 \mathrm{e}-04$ \\
\hline & 1.536 & 1.536 & 1.561 & 1.561 & 1.615 & 1.615 & 1.653 & 1.653 \\
\hline \multirow[t]{2}{*}{$10^{-7}$} & $2.372 \mathrm{e}-02$ & $2.372 \mathrm{e}-02$ & $8.177 \mathrm{e}-03$ & $8.177 \mathrm{e}-03$ & $2.770 \mathrm{e}-03$ & $2.770 \mathrm{e}-03$ & $9.040 \mathrm{e}-04$ & $9.040 \mathrm{e}-04$ \\
\hline & 1.536 & 1.536 & 1.561 & 1.561 & 1.615 & 1.615 & 1.653 & 1.653 \\
\hline \multirow[t]{2}{*}{$10^{-8}$} & $2.372 \mathrm{e}-02$ & $2.372 \mathrm{e}-02$ & $8.177 \mathrm{e}-03$ & $8.177 \mathrm{e}-03$ & $2.770 \mathrm{e}-03$ & $2.770 \mathrm{e}-03$ & $9.040 \mathrm{e}-04$ & $9.040 \mathrm{e}-04$ \\
\hline & 1.536 & 1.536 & 1.561 & 1.561 & 1.615 & 1.615 & 1.653 & 1.653 \\
\hline$e^{N}$ & $2.372 \mathrm{e}-2$ & $2.372 \mathrm{e}-02$ & $8.177 \mathrm{e}-03$ & $8.177 \mathrm{e}-03$ & $2.770 \mathrm{e}-03$ & $2.770 \mathrm{e}-03$ & $9.042 \mathrm{e}-04$ & $1.221 \mathrm{e}-03$ \\
\hline$p^{N}$ & 1.536 & 1.536 & 1.561 & 1.561 & 1.615 & 1.181 & 1.632 & 0.997 \\
\hline
\end{tabular}

Example 3. Consider the following BVP of the form (1.1)-(1.2):

$$
\left\{\begin{array}{l}
\varepsilon u^{\prime \prime}(x)-(1+x(0.5-x)) u^{\prime}(x)-x(1-x) u(x)=-(1+2 x), x \in(0,0.5), \\
\varepsilon u^{\prime \prime}(x)+(1+x(x-0.5)) u^{\prime}(x)-x(1-x) u(x)=(1+2 x), x \in(0.5,1), \\
{[u](0.5)=0, \quad[d u / d x](0.5)=0,} \\
u(0)=0, \quad u(1)=0 .
\end{array}\right.
$$

In Figure 3 we display the respective numerical solutions $U$ of the BVPs (6.2) and (6.3) computed using the newly proposed scheme (3.1)-(3.3), which clearly shows the presence of interior layers near the point of discontinuity $x=0.5$ as the parameter $\varepsilon$ decreases.

As the exact solution of the BVPs (6.2) and (6.3) are not known, in order to compute the maximum point-wise error $E_{\varepsilon}^{N}$ and the corresponding order of convergence $P_{\varepsilon}^{N}$ for Examples 2 and 3, we use the following interpolation technique.

Let $U^{N}$ denote the numerical solution obtained on the mesh $\bar{\Omega}^{N}$ and $\bar{U}^{4096}$ denote the linear interpolation to $U^{N}$ on the mesh $\bar{\Omega}^{N}$. Then for each $\varepsilon$, we calculate the maximum point-wise error by

$$
E_{\varepsilon}^{N}=\max _{x_{i} \in \bar{\Omega}^{N}}\left|U_{i}^{N}-\bar{U}_{i}^{4096}\right|
$$




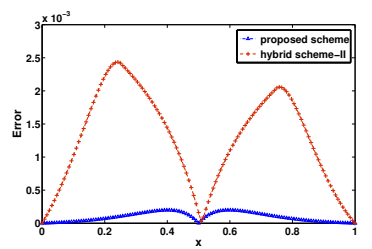

(a) $\varepsilon=10^{-1}$

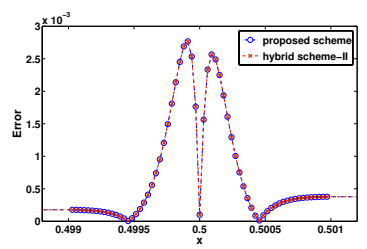

(b) $\varepsilon=10^{-4}$.

Figure 2. Comparison of error $=\left|u-U^{N}\right|$ obtained for $\varepsilon=10^{-1}, 10^{-4}$ and $N=128$ on the mesh $\bar{\Omega}^{N}$, for Example 1. In part (b) the zoomed view near $x=0.5$ is presented.

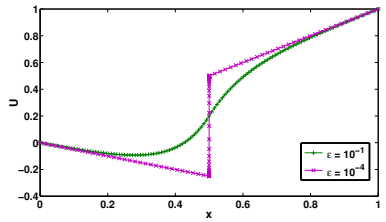

a)

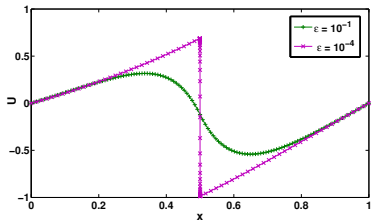

b)

Figure 3. Numerical solutions for: a) Example 2 on the mesh $\bar{\Omega}^{N}$ for $N=128$, b) Example 3 on the mesh $\bar{\Omega}^{N}$ for $N=128$.

and the corresponding order of convergence by $P_{\varepsilon}^{N}=\log _{2}\left(E_{\varepsilon}^{N} / E_{\varepsilon}^{2 N}\right)$. Now, for each $N$, we define $E^{N}=\max E_{\varepsilon}^{N}$ as the $\varepsilon$-uniform maximum point-wise error and $P^{N}=\log _{2}\left(E^{N} / E^{2 N}\right)^{\varepsilon}$ as the corresponding $\varepsilon$-uniform order of convergence.

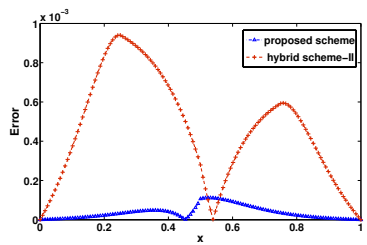

(a) $\varepsilon=10^{-1}$

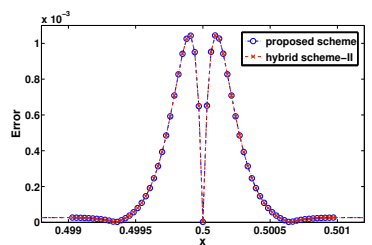

(b) $\varepsilon=10^{-4}$

Figure 4. Comparison of error $=\left|U^{N}-\bar{U}^{4096}\right|$ obtained for $\varepsilon=10^{-1}, 10^{-4}$ and $N=128$ on the mesh $\bar{\Omega}^{N}$, for Example 2. In part (b) the zoomed view near $x=0.5$ is presented.

For various values of $\varepsilon$ and $N$, the maximum point-wise errors $E_{\varepsilon}^{N}$ and the corresponding order of convergence $P_{\varepsilon}^{N}$ computed using the newly proposed scheme (3.1)-(3.3) and the hybrid scheme-II are presented in Tables 2 and 3, respectively for Examples 2 and 3 . We also display the $\varepsilon$-uniform errors $E^{N}$ and the corresponding order of convergence $P^{N}$ computed using both the hybrid schemes in Tables 2 and 3, respectively for Examples 2 and 3.

In spite of this, for clarity of the presentation, the errors associated with the numerical solutions of the BVPs (6.2) and (6.3) for $\varepsilon=10^{-1}, 10^{-4}$ computed 
Table 2. Maximum point-wise errors and order of convergence for Example 2.

\begin{tabular}{|c|c|c|c|c|c|c|c|c|}
\hline \multirow[t]{3}{*}{$\varepsilon$} & \multicolumn{8}{|c|}{ Number of mesh intervals, $N$} \\
\hline & \multicolumn{2}{|c|}{32} & \multicolumn{2}{|c|}{64} & \multicolumn{2}{|c|}{128} & \multicolumn{2}{|c|}{256} \\
\hline & $\begin{array}{l}\text { new } \\
\text { scheme }\end{array}$ & $\begin{array}{l}\text { scheme } \\
\text { II }\end{array}$ & $\begin{array}{l}\text { new } \\
\text { scheme }\end{array}$ & $\begin{array}{l}\text { scheme } \\
\text { II }\end{array}$ & $\begin{array}{l}\text { new } \\
\text { scheme }\end{array}$ & $\begin{array}{l}\text { scheme } \\
\text { II }\end{array}$ & $\begin{array}{l}\text { new } \\
\text { scheme }\end{array}$ & $\begin{array}{l}\text { scheme } \\
\text { II }\end{array}$ \\
\hline \multirow[t]{2}{*}{1} & $8.837 \mathrm{e}-05$ & $4.944 \mathrm{e}-04$ & $2.235 \mathrm{e}-05$ & $2.267 \mathrm{e}-04$ & $5.619 \mathrm{e}-06$ & $1.071 \mathrm{e}-04$ & $1.405 \mathrm{e}-06$ & $5.075 \mathrm{e}-05$ \\
\hline & 1.982 & 1.124 & 1.992 & 1.082 & 1.999 & 1.077 & 2.014 & 1.114 \\
\hline \multirow[t]{2}{*}{$10^{-1}$} & $1.571 \mathrm{e}-03$ & $3.875 \mathrm{e}-03$ & $4.260 \mathrm{e}-04$ & $1.914 \mathrm{e}-03$ & $1.116 \mathrm{e}-04$ & $9.403 \mathrm{e}-04$ & $2.859 \mathrm{e}-05$ & $4.550 \mathrm{e}-04$ \\
\hline & 1.883 & 1.017 & 1.932 & 1.025 & 1.964 & 1.047 & 1.996 & 1.099 \\
\hline \multirow[t]{2}{*}{$10^{-2}$} & $9.256 \mathrm{e}-03$ & $9.256 \mathrm{e}-03$ & $3.202 \mathrm{e}-03$ & $3.202 \mathrm{e}-03$ & $1.096 \mathrm{e}-03$ & $1.086 \mathrm{e}-03$ & $3.579 \mathrm{e}-04$ & $3.568 \mathrm{e}-04$ \\
\hline & 1.531 & 1.531 & 1.545 & 1.560 & 1.615 & 1.605 & 1.700 & 1.698 \\
\hline \multirow[t]{2}{*}{$10^{-3}$} & $9.055 \mathrm{e}-03$ & $9.055 \mathrm{e}-03$ & $3.122 \mathrm{e}-03$ & $3.122 \mathrm{e}-03$ & $1.048 \mathrm{e}-03$ & $1.048 \mathrm{e}-03$ & $3.429 \mathrm{e}-04$ & $3.429 \mathrm{e}-04$ \\
\hline & 1.536 & 1.536 & 1.573 & 1.573 & 1.612 & 1.612 & 1.693 & 1.693 \\
\hline \multirow[t]{2}{*}{$10^{-4}$} & $9.032 \mathrm{e}-03$ & $9.032 \mathrm{e}-03$ & $3.112 \mathrm{e}-03$ & $3.112 \mathrm{e}-03$ & $1.044 \mathrm{e}-03$ & $1.044 \mathrm{e}-03$ & $3.414 \mathrm{e}-04$ & $3.413 \mathrm{e}-04$ \\
\hline & 1.537 & 1.537 & 1.575 & 1.575 & 1.612 & 1.613 & 1.690 & 1.691 \\
\hline \multirow[t]{2}{*}{$10^{-5}$} & $9.030 \mathrm{e}-03$ & $9.030 \mathrm{e}-03$ & $3.111 \mathrm{e}-03$ & $3.111 \mathrm{e}-03$ & $1.044 \mathrm{e}-03$ & $1.044 \mathrm{e}-03$ & $3.415 \mathrm{e}-04$ & $3.415 \mathrm{e}-04$ \\
\hline & 1.537 & 1.537 & 1.575 & 1.575 & 1.612 & 1.612 & 1.688 & 1.688 \\
\hline \multirow[t]{2}{*}{$10^{-6}$} & $9.029 \mathrm{e}-03$ & $9.029 \mathrm{e}-03$ & $3.110 \mathrm{e}-03$ & $3.110 \mathrm{e}-03$ & $1.043 \mathrm{e}-03$ & $1.043 \mathrm{e}-03$ & $3.410 \mathrm{e}-04$ & $3.408 \mathrm{e}-04$ \\
\hline & 1.537 & 1.537 & 1.575 & 1.576 & 1.613 & 1.614 & 1.691 & 1.694 \\
\hline \multirow[t]{2}{*}{$10^{-7}$} & $9.029 \mathrm{e}-03$ & $9.029 \mathrm{e}-03$ & $3.110 \mathrm{e}-03$ & $3.110 \mathrm{e}-03$ & $1.043 \mathrm{e}-03$ & $1.043 \mathrm{e}-03$ & $3.410 \mathrm{e}-04$ & $3.410 \mathrm{e}-04$ \\
\hline & 1.537 & 1.537 & 1.575 & 1.575 & 1.613 & 1.613 & 1.691 & 1.691 \\
\hline \multirow[t]{2}{*}{$10^{-8}$} & $9.029 \mathrm{e}-03$ & $9.029 \mathrm{e}-03$ & $3.110 \mathrm{e}-03$ & $3.110 \mathrm{e}-03$ & $1.043 \mathrm{e}-03$ & $1.043 \mathrm{e}-03$ & $3.410 \mathrm{e}-04$ & $3.408 \mathrm{e}-04$ \\
\hline & 1.537 & 1.537 & 1.575 & 1.576 & 1.613 & 1.614 & 1.692 & 1.694 \\
\hline$E^{N}$ & $9.256 \mathrm{e}-03$ & $9.256 \mathrm{e}-03$ & $3.202 \mathrm{e}-03$ & $3.202 \mathrm{e}-03$ & $1.096 \mathrm{e}-03$ & $1.086 \mathrm{e}-03$ & $3.579 \mathrm{e}-04$ & $4.550 \mathrm{e}-04$ \\
\hline$P^{N}$ & 1.531 & 1.531 & 1.545 & 1.560 & 1.615 & 1.255 & 1.700 & 1.099 \\
\hline
\end{tabular}

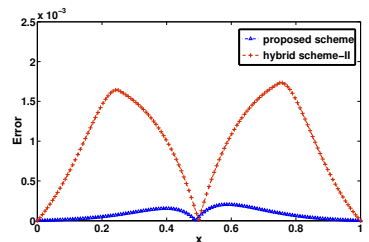

(a) $\varepsilon=10^{-1}$

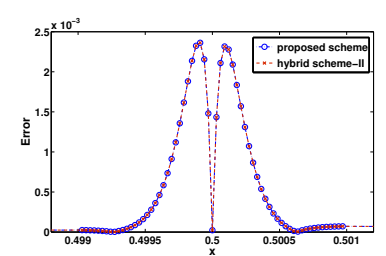

(b) $\varepsilon=10^{-4}$

Figure 5. Comparison of error $=\left|U^{N}-\bar{U}^{4096}\right|$ obtained for $\varepsilon=10^{-1}, 10^{-4}$ and $N=128$ on the mesh $\bar{\Omega}^{N}$, for Example 3. In part (b) the zoomed view near $x=0.5$ is presented.

using both the hybrid schemes are depicted, respectively in Figures 4 and 5.

\subsection{Observations and concluding remarks}

From the numerical experiments, we made the following observations about the newly proposed scheme for solving a class of singularly perturbed BVPs of the form (1.1)-(1.2):

- It is observed that the $\varepsilon$-uniform errors (i.e., $e^{N}$ or $E^{N}$ ) obtained in Tables 1, 2 and 3, decrease monotonically as $N$ increases. This ensures that the newly proposed scheme is $\varepsilon$-uniformly convergent. 
Table 3. Maximum point-wise errors and order of convergence for Example 3.

\begin{tabular}{|c|c|c|c|c|c|c|c|c|}
\hline \multirow[t]{3}{*}{$\varepsilon$} & \multicolumn{8}{|c|}{ Number of mesh intervals, $N$} \\
\hline & \multicolumn{2}{|c|}{32} & \multicolumn{2}{|c|}{64} & \multicolumn{2}{|c|}{128} & \multicolumn{2}{|c|}{256} \\
\hline & $\begin{array}{l}\text { new } \\
\text { scheme }\end{array}$ & $\begin{array}{l}\text { scheme } \\
\text { II }\end{array}$ & $\begin{array}{l}\text { new } \\
\text { scheme }\end{array}$ & $\begin{array}{l}\text { scheme } \\
\text { II }\end{array}$ & $\begin{array}{l}\text { new } \\
\text { scheme }\end{array}$ & $\begin{array}{l}\text { scheme } \\
\text { II }\end{array}$ & $\begin{array}{l}\text { new } \\
\text { scheme }\end{array}$ & $\begin{array}{l}\text { scheme } \\
\text { II }\end{array}$ \\
\hline \multirow[t]{2}{*}{1} & $2.434 \mathrm{e}-04$ & $1.225 \mathrm{e}-03$ & $6.123 \mathrm{e}-05$ & $5.635 \mathrm{e}-04$ & $1.534 \mathrm{e}-05$ & $2.663 \mathrm{e}-04$ & $3.829 \mathrm{e}-06$ & $1.262 \mathrm{e}-04$ \\
\hline & 1.990 & 1.120 & 1.996 & 1.081 & 2.002 & 1.077 & 2.016 & 1.114 \\
\hline \multirow[t]{2}{*}{$10^{-1}$} & $3.273 \mathrm{e}-03$ & $6.858 \mathrm{e}-03$ & $8.143 \mathrm{e}-04$ & $3.480 \mathrm{e}-03$ & $2.036 \mathrm{e}-04$ & $1.733 \mathrm{e}-03$ & $5.078 \mathrm{e}-05$ & $8.451 \mathrm{e}-04$ \\
\hline & 2.007 & 0.978 & 1.999 & 1.005 & 2.003 & 1.036 & 2.016 & 1.094 \\
\hline \multirow[t]{2}{*}{$10^{-2}$} & $2.132 \mathrm{e}-02$ & $2.131 \mathrm{e}-02$ & $7.314 \mathrm{e}-03$ & $7.313 \mathrm{e}-03$ & $2.448 \mathrm{e}-03$ & $2.447 \mathrm{e}-03$ & $8.013 \mathrm{e}-04$ & $8.006 \mathrm{e}-04$ \\
\hline & 1.543 & 1.543 & 1.578 & 1.579 & 1.611 & 1.612 & 1.717 & 1.687 \\
\hline \multirow[t]{2}{*}{$10^{-3}$} & $2.074 \mathrm{e}-02$ & $2.074 \mathrm{e}-02$ & $7.109 \mathrm{e}-03$ & $7.108 \mathrm{e}-03$ & $2.376 \mathrm{e}-03$ & $2.375 \mathrm{e}-03$ & $7.754 \mathrm{e}-04$ & $7.751 \mathrm{e}-04$ \\
\hline & 1.545 & 1.545 & 1.581 & 1.581 & 1.615 & 1.615 & 1.691 & 1.692 \\
\hline \multirow[t]{2}{*}{$10^{-4}$} & $2.067 \mathrm{e}-02$ & $2.067 \mathrm{e}-02$ & $7.076 \mathrm{e}-03$ & $7.076 \mathrm{e}-03$ & $2.362 \mathrm{e}-03$ & $2.362 \mathrm{e}-03$ & $7.695 \mathrm{e}-04$ & $7.696 \mathrm{e}-04$ \\
\hline & 1.546 & 1.546 & 1.582 & 1.582 & 1.618 & 1.618 & 1.695 & 1.695 \\
\hline \multirow[t]{2}{*}{$10^{-5}$} & $2.066 \mathrm{e}-02$ & $2.066 \mathrm{e}-02$ & 7.072e-03 & $7.072 \mathrm{e}-03$ & $2.360 \mathrm{e}-03$ & $2.360 \mathrm{e}-03$ & $7.687 \mathrm{e}-04$ & $7.684 \mathrm{e}-04$ \\
\hline & 1.546 & 1.546 & 1.583 & 1.583 & 1.618 & 1.619 & 1.696 & 1.697 \\
\hline \multirow[t]{2}{*}{$10^{-6}$} & $2.066 \mathrm{e}-02$ & $2.066 \mathrm{e}-02$ & $7.072 \mathrm{e}-03$ & $7.072 \mathrm{e}-03$ & $2.360 \mathrm{e}-03$ & $2.360 \mathrm{e}-03$ & $7.682 \mathrm{e}-04$ & 7.681e-04 \\
\hline & 1.546 & 1.546 & 1.583 & 1.583 & 1.619 & 1.619 & 1.698 & 1.698 \\
\hline \multirow[t]{2}{*}{$10^{-7}$} & $2.066 \mathrm{e}-02$ & $2.066 \mathrm{e}-02$ & $7.074 \mathrm{e}-03$ & $7.074 \mathrm{e}-03$ & $2.363 \mathrm{e}-03$ & $2.363 \mathrm{e}-03$ & $7.715 \mathrm{e}-04$ & $7.712 \mathrm{e}-04$ \\
\hline & 1.546 & 1.546 & 1.581 & 1.582 & 1.615 & 1.615 & 1.686 & 1.687 \\
\hline \multirow[t]{2}{*}{$10^{-8}$} & $2.066 \mathrm{e}-02$ & $2.066 \mathrm{e}-02$ & 7.073e-03 & $7.072 \mathrm{e}-03$ & $2.361 \mathrm{e}-03$ & $2.360 \mathrm{e}-03$ & $7.694 \mathrm{e}-04$ & $7.684 \mathrm{e}-04$ \\
\hline & 1.546 & 1.546 & 1.582 & 1.583 & 1.617 & 1.619 & 1.694 & 1.697 \\
\hline$E^{N}$ & $2.132 \mathrm{e}-02$ & $2.131 \mathrm{e}-02$ & $7.314 \mathrm{e}-03$ & $7.313 \mathrm{e}-03$ & $2.448 \mathrm{e}-03$ & $2.447 \mathrm{e}-03$ & $8.013 \mathrm{e}-04$ & $8.451 \mathrm{e}-04$ \\
\hline$P^{N}$ & 1.543 & 1.543 & 1.578 & 1.579 & 1.611 & 1.534 & 1.717 & 1.094 \\
\hline
\end{tabular}

- On the other hand, from the numerical results displayed in Tables 1, 2 and 3 , it is clear that the $\varepsilon$-uniform order of convergence of the hybrid scheme-II is nearly one as $N$ increases, whereas the proposed hybrid scheme converges $\varepsilon$-uniformly with almost second-order accuracy. These observations are in excellent agreement with the theoretical results obtained for the proposed hybrid scheme and the hybrid scheme-II. As a complement of these observations, the $\varepsilon$-uniform errors computed using both the hybrid schemes for Examples 1, 2 and 3 are plotted in Figure 6.

- More precisely, we notice that the current hybrid scheme yields higherorder accurate numerical results, particularly for larger values of $\varepsilon$ (e.g., $\varepsilon=10^{-1}$ ), in comparison with the hybrid scheme-II; although the maximum point-wise errors caused by both the hybrid schemes for the smaller values of $\varepsilon$ (e.g., $\varepsilon=10^{-4}$ ) decrease with same order of accuracy as $N$ increases.

Therefore, based on the above mentioned observations we can conclude that the newly proposed scheme is at worst almost second-order accurate, whereas the hybrid scheme-II is at worst first-order accurate, irrespective of the perturbation parameter $\varepsilon$, which reflects a significant improvement in the current hybrid scheme in comparison with the hybrid scheme-II. 


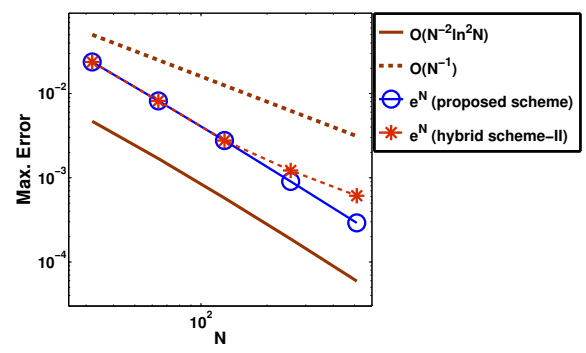

(a) Example 1.

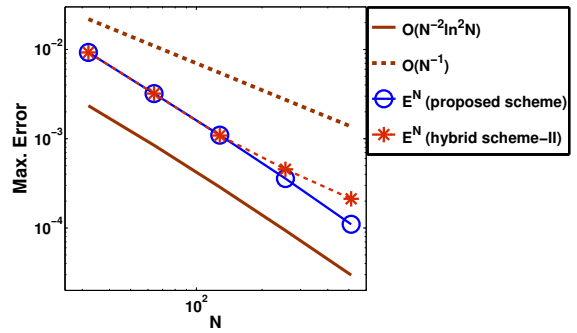

(b) Example 2 .

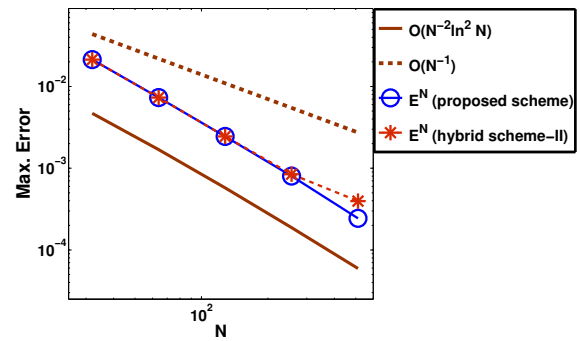

(c) Example 3 .

Figure 6. Loglog plot of $\varepsilon$-uniform maximum point-wise errors.

\section{References}

[1] Z. Cen. A hybrid difference scheme for a singularly perturbed convection-diffusion problem with discontinuous convection coefficient. Applied Mathematics and Computation, 169(1):689-699, 2005. https://doi.org/10.1016/j.amc.2004.08.051.

[2] P.A. Farrell, A.F. Hegarty, J.J.H. Miller, E. O'Riordan and G.I. Shishkin. Global maximum norm parameter-uniform numerical method for a singularly perturbed convection-diffusion problem with discontinuous convection coefficient. Mathematical and Computer Modelling, 40(11):1375-1392, 2004. https://doi.org/10.1016/j.mcm.2005.01.025.

[3] P.A. Farrell, A.F. Hegarty, J.J.M. Miller, E. O'Riordan and G.I. Shishkin. Robust 
Computational Techniques for Boundary Layers. Chapman \& Hall/CRC Press, 2000.

[4] P.A. Farrell, E. O'Riordan and G.I. Shishkin. A class of singularly perturbed quasilinear differential equations with interior layers. Mathematics of Computation, 78(265):103-127, 2009. https://doi.org/10.1090/S0025-5718-08-02157-1.

[5] M.K. Kadalbajoo and V. Gupta. A brief survey on numerical methods for solving singularly perturbed problems. Applied Mathematics and Computation, 217(8):3641-3716, 2010. https://doi.org/10.1016/j.amc.2010.09.059.

[6] J.J.H. Miller, E. O'Riordan and G.I. Shishkin. Fitted Numerical Methods for Singular Perturbation Problems. World Scientific, Singapore, 1996.

[7] K. Mukherjee and S. Natesan. Optimal error estimate of upwind scheme on Shishkin-type meshes for singularly perturbed parabolic problems with discontinuous convection coefficients. BIT Numerical Mathematics, 51(2):289-315, 2011. https://doi.org/10.1007/s10543-010-0292-2.

[8] K. Mukherjee and S. Natesan. $\varepsilon$-Uniform error estimate of hybrid numerical scheme for singularly perturbed parabolic problems with nterior layers. Numerical Algorithms, 58(1):103-141, 2011. https://doi.org/10.1007/s11075-011-94496.

[9] E. O'Riordan and G.I. Shishkin. Singularly perturbed parabolic problems with non-smooth data. Journal of Computational and Applied Mathematics, 166(1):233-245, 2004. https://doi.org/10.1016/j.cam.2003.09.025.

[10] R.M. Priyadharshini and N. Ramanujam. A hybrid difference scheme for singularly perturbed second order ordinary differential equations with discontinuous convection coefficient and mixed type boundary conditions. International Journal of Computational Methods, 05(04):575-593, 2008. https://doi.org/10.1142/S0219876208001637.

[11] H.-G. Roos, M. Stynes and L. Tobiska. Robust Numerical Methods for Singularly Perturbed Differential Equations. Springer-Verlag, Berlin, Heidelberg, 2008. https://doi.org/10.1007/978-3-540-34467-4.

[12] V. Shanthi, N. Ramanujam and S. Natesan. Fitted mesh method for singularly perturbed reaction convection-diffusion problems with boundary and interior layers. Journal of Applied Mathematics and Computing, 22(1):49-65, 2006. https://doi.org/10.1007/BF02896460. 